\title{
Der Traum vom leeren Raum: Perspektiven einer performativen Lehr-, Lern- und Forschungskultur
}

\section{Manfred Schewe}

Im Folgenden beziehe ich mich schlaglichtartig auf einige Aspekte meines Plenarvortrags.

Leitfragen, die dem Vortrag zugrunde lagen -

- Seit wann ist in pädagogischen Kontexten von ,performativ' die Rede und welche Bedeutungsschichten hat dieser Begriff? Inwiefern lässt sich in verschiedenen Disziplinen bereits von einer lebendigen Vielfalt und auch theoretischen Fundierung ,performativer Praxis'sprechen?

- Wie können wir dazu beitragen, dass in den 2020er Jahren - idealerweise im Rahmen einer ,Performativen Hochschuldidaktik ${ }^{\star}$ - in verschiedenen Disziplinen Konzepte einer performativen Didaktik (weiter) entwickelt werden?

Über die in den Leitfragen enthaltene inhaltliche Ebene hinaus war mir die Form des Vortrags wichtig. Zu den ,performativen Elementen', die den Vortrag durchzogen, gehörte eine Geschichte, auf die ich beim Lesen von Peter Brooks (1988) The Shifting Point. Forty years of theatrical exploration 1946-1987 aufmerksam geworden war und die so beginnt:

God, seeing how desperately bored everyone was on the seventh day of creation, racked his overstretched imagination to find something more to add to the completeness he had just conceived. Suddenly his inspiration burst even beyond his own limitless bounds and he saw a further aspect of reality: its possibility to imitate itself. So he invented theatre.

Während meiner Erzählung gab es Punkte, an denen ich die SymposiumTeilnehmer*innen dazu aufforderte, spontan an der Konstruktion der Geschichte mitzuwirken; etwa an dem Punkt, an dem Gott erklärt, dass das Theater Trost für den Trinker und für die Einsamen sein kann. „Für wen sonst kann das Theater wichtig sein und warum?" lautete meine Aufforderung, auf die vom Publikum erfreulich schnell reagiert wurde.

Peter Brooks Geschichte wird in dieser Ausgabe abgedruckt, so können Scenario-Leser*innen nachlesen, dass es dem bekannten Theaterregisseur 
darin insbesondere um die Botschaft geht, dass Theater für alle im Publikum INTERESSANT sein muss. Gilt nicht das Gleiche für den Unterricht? Sollte dieser nicht für alle in der Schulklasse bzw. im Uni-Seminar interessant sein? Wenn man bedenkt, was alles nötig ist, um ein Stück so auf die Bühne zu bringen, dass es für alle im Publikum interessant ist: Ist das nicht geradezu eine didaktische Meisterleistung?

Eine räumliche Perspektive - Mit dieser Geschichte hatte ich die Aufmerksamkeit der Symposium-Teilnehmer*innen auf die Kunstform Theater gerichtet. Um besondere Merkmale dieser Kunstform geht es Peter Brook in seiner Veröffentlichung The Empty Space (1990), die mit dem Satz beginnt:

I can take any empty space and call it a bare stage. A man walks across this empty space whilst someone else is watching him, and this is all that is needed for an act of theatre to be engaged.

Mit dem Verweis auf dieses Zitat war eine Anknüpfung an den Titel meines Vortrags erfolgt. Auf meine Nachfrage hin bestätigten einige SymposiumTeilnehmer*innen, dass Unterrichtsräume an ihren Institutionen oft keine Bewegungsfreiheit lassen und sich daher für performatives Lehren und Lernen nicht besonders eignen; man solle daher darauf hinwirken, dass in der jeweiligen Institution zweckdienliche Lernumgebungen geschaffen werden und der Traum vom leeren Raum Wirklichkeit werde (vgl. zu diesem Thema auch die Internationale Scenario Forum Konferenz Performative Spaces in Language, Literature and Culture Education - University College Cork 2017).

Mit dem folgenden Foto rief ich in Erinnerung, dass es Verbindungslinien zwischen Theater und Lehre (vgl. hierzu die Darstellung in Schewe 2013) schon lange gibt.

Diese historische Perspektive erweiterte ich u.a. durch einen Bezug auf

- Johann Wolfgang von Goethes Bildungsroman Wilhelm Meisters Lehrjahre (1795/96), in dem die starke Wirkung beschrieben wird, die Theater auf den jungen Wilhelm hat. Dazu gehört etwa ein bewussterer Umgang mit seinem Körper, die Überwindung seiner Scheu und damit von Auftrittsängsten, eine größere Gewandtheit im sozialen Umgang, größere Wendigkeit in öffentlichen Kontexten und nicht zuletzt die Erweiterung seiner sprachbezogenen Kompetenzen. Für Wilhelms Selbstfindung und Persönlichkeitsbildung wird Theater zu einem wichtigen Erfahrungsraum.

- Friedrich Schillers Gedicht An die Freunde (1879), in dem die Rede ist von ,Brettern, die die Welt bedeuten'. Bemerkenswert ist die Wichtigkeit, die der idealistische Dichter der Phantasietätigkeit zumisst. Mit einfachsten Mitteln, auf lediglich ein paar Brettern sozusagen, lassen sich die engen Grenzen von Alltagserfahrung imaginativ überwinden. Denn der Raum der Fiktion ist grenzenlos und darin lässt sich - als Gegenentwurf zur empfundenen Lebensenge - eine alternative Welt erschaffen. 
Eine ästhetische Perspektive - Schillers Gedicht wurde zum Impuls für eine praktische Übung. Die Symposium-Teilnehmer*innen wurden aufgefordert, sich folgende Situation vorzustellen:

Stelle Dir vor, Du bist in Deinem Zimmer und liegst auf dem Bett. Endlich, es ist Abend. Du hast weiter keine Verpflichtung mehr heute und kannst entspannen, die Seele baumeln lassen. Du nimmst Dir das Buch, das auf dem Nachttisch liegt und beginnst darin zu lesen. Nach einer Weile unterbrichst Du die Lektüre kurz. Von irgendwoher scheint ein ungewöhnlicher Geruch zu kommen. Aber Du denkst Dir nichts weiter dabei und liest weiter. Und jetzt hörst Du ein Geräusch - so als würde etwas Großes geschoben. Du blickst auf und in genau diesem Moment öffnet sich die Tür zu Deinem Zimmer und vor Dir steht ein grüner Drache. Was machst Du?

Ich forderte alle auf, spontan ihre jeweils individuelle Reaktion aufzuschreiben. Anschließend sollten sich die Teilnehmer*innen zu zweit zusammentun und einander erzählen, sie sie auf die ungewöhnliche Situation reagiert hatten. Es kam zu einem sehr lebendigen Austausch, anhand der unterschiedlichen Reaktionen speziell auch über verschiedene Formen des Umgangs mit dem Fremden. Die Diskussion kann hier nicht im Einzelnen wiedergegeben werden, verwiesen sei aber auf den Artikel von Beug/Schewe (1999), in dem das Experiment mit dem grünen Drachen ausführlicher beschrieben wird.

Die folgende Übung sollte klären helfen, welche Bedeutungsschichten das Wort ,performativ' hat. Dabei kam auch die Imagination wieder ins Spiel ...

Eine theoretisch-terminologische Perspektive -Um dieSymposium-Teilnehmer*innen zu aktiveren, zeigte ich ihnen eine PowerPoint-Folie, auf der das Wort PERFORMATIV, vertikal buchstabiert' ${ }^{\prime}$ war. Ich forderte sie auf, Wörter zu nennen, die mit dem jeweiligen Buchstaben beginnen und sich mit der Essenz des Performativen assoziieren lassen. Die spontanen Nennungen können hier nicht rekonstruiert werden, in der grafischen Darstellung unten finden sich aber meine eigenen Assoziationen, die ich allen nach dem gemeinsamen Brainstorming zeigte:

Diese Übung sollte dazu anregen, sich mit den Bedeutungsebenen des Begriffs ,performativ' auseinanderzusetzen. Aus der Perspektive des Sprachphilosophen Austin (1962) verweist der Begriff auf das Handeln durch Sprache; in einem weiteren (künstlerisch orientierten) Sinne bezieht er sich auf Formen kreativen Handelns. Beachtenswert ist, dass im Begriff ,performativ' der Aspekt persönlicher Entwicklung (,performativ ${ }^{c}$ ) und der Aspekt Form (,performativ') betont wird.

In der grafischen Darstellung wird hervorgehoben, dass im performativ gestalteten Unterricht besonderer Wert auf die ästhetische Form gelegt wird, d.h. auf Imagination, sinnliche Anschauung (Aisthesis), Emotion und auf die Reflexion ästhetischer Erfahrung.

Diese Überlegungen können hier nicht weiter ausgeführt werden, verwiesen sei aber auf weitere Quellen, in denen eine intensivere Auseinandersetzung mit dem Begriff,performativ ${ }^{`}$ bzw. dem Konzept performativen Lehrens und Lernens 
stattfindet: in Bezug auf die performative Wende in den Kulturwissenschaften siehe z.B. Bachmann-Medick (2010); aus theaterwissenschaftlicher Perspektive z.B. Fischer-Lichte (2004, 2012); in Bezug auf Aspekte performativen Lehrens und Lernens z.B. Even \& Schewe (2016), Hudelist \& Krammer (2017), Mentz \& Fleiner (2018). An meinen Plenarvortrag inhaltlich gekoppelt war ein Thesenpapier, das verdeutlichen sollte, was speziell unter einer ,performativen Lehr-, Lern- und Forschungskultur' zu verstehen ist. Zum Ende der Tagung wurden die Thesen engagiert diskutiert und die Diskussionsergebnisse wurden vom Scenario Forum Organisationsteam zu ,Empfehlungen' gebündelt (siehe den Beitrag von Jogschies, Schewe \& Stöver-Blahak in dieser Ausgabe).

Eine überraschende Perspektive — Wie anfangs erwähnt, hatte ich mich um eine performative Gestaltung meines Plenarvortrags bemüht. Hierzu abschließend ein weiteres Beispiel: Ich verteilte an alle Symposium-Teilnehmer*innen Karteikarten und vermerkte darauf, in welcher Weise sie sich an der bevorstehenden praktischen Übung beteiligen sollten. Eine Person im Publikum bekam eine JOKER-Karte, auf der es hieß: „Niemand im Raum weiß, dass du diese JOKER-Karte gezogen hast. Im Laufe der nächsten 40 Minuten wirst du in einem von dir bestimmten Moment - den Vortrag von Manfred Schewe unterbrechen, indem du im Tagungsraum etwas Ungewöhnliches machst."

Als ich gerade dabei war, ein längeres Zitat $\mathrm{zu}$ vorzulesen, stand eine Teilnehmerin aus der zweiten Reihe rechts auf und bewegte sich in meine Richtung. Sie ging auf das große Blumengesteck zu, das vor meinem Rednerpult stand. Sie fing an, dieses hin und her zu schieben. Ich machte einfach weiter mit dem Vorlesen des Zitats, bemerkte aber, dass die Aufmerksamkeit der Symposium-Teilnehmer*innen nicht mehr auf mich gerichtet war, sondern auf das ,phänomenale Geschehen': Die Teilnehmerin hob nun mit beiden Händen sogar das große Blumengesteck auf, bewegte sich in eine andere Ecke des Raumes, deponierte es dort und begann es neu zu arrangieren, um sich nach einer Weile gemächlich wieder auf ihren Platz zu begeben.

Was war geschehen? Ein routinierter Ablauf - jemand trägt vor und die Zuhörer*innen hören zu -war unterbrochen worden (in diesem Zusammenhang siehe auch Campbell 2017). Dieses ,Ereignis' zeigte eine besondere Wirkung; das überraschte Organisationsteam jedenfalls schien nicht so recht zu wissen, was von der Teilnehmerin, die mit dem Blumengesteck hantierte, zu halten sei und war kurz davor einzugreifen; etliche aus dem Publikum warfen sich Blicke zu und ich hörte Gelächter ...

Was ich auf diese Weise demonstrieren wollte: Performativer Unterricht ist oft ,ereignishaft', spielt mit dem Unerwarteten.

Abschließend sei noch angemerkt, dass das Blumengesteck während der Tagung immer wieder zum Bezugspunkt wurde. Die Teilnehmer*innen erwähnten das Gesteck in Gesprächen, benutzten es als Requisit in den praktischen Workshop-Übungen bzw. auch als Kopfschmuck, als sich die Teilnehmer*innen zum Ende der Tagung für ein szenisches Erinnerungsfoto (siehe am Ende des Kurzfilms) in Stellung brachten. Warum hatte das Blumengesteck eine so nach- 
haltige Wirkung und sich so stark in das Gedächtnis von allen eingegraben? Eine Frage, die zum anschließenden Vortrag und Workshop Und Action! Vom Hirn ins Herz bis in die Füße von Michaela Sambanis und Maik Walter überleitete.

\section{Bibliografie}

Austin, John Langshaw (1962): How to do things with words. Oxford: Clarendon

Bachmann-Medick, Doris (2010): Cultural Turns. Neuorientierungen in den Kulturwissenschaften. Reinbek bei Hamburg: Rowohlt

Beug, Joachim \& Schewe, Manfred (1999): Learning and teaching culture from an aesthetic perspective: some theoretical reflections and practical examples. In: Chambers, Angela \& Ó Baoill, Dónall P. (Hrsg.): Intercultural Communication and Language Learning. Dublin: The Irish Association for Applied Linguistics (IRAAL) in association with the Royal Irish Academy, 119-134

Brook, Peter (1988): The Shifting Point. Forty years of theatrical exploration 1946-1987. London: Methuen

Brook, Peter (1990): The Empty Space. London: Penguin

Campbell, Lee (2017): Collaborators and Hecklers: Performative Pedagogy and Interruptive Processes. In: Scenario XI/1, 33-64

Even, Susanne \& Schewe, Manfred (Hrsg.) (2016): Performatives Lehren, Lernen und Forschen - Performative Teaching, Learning, Research. Berlin: Schibri

Fischer-Lichte, Erika (2012): Performativität. Eine Einführung. Bielefeld: transcript Verlag

Fischer-Lichte, Erika (2004): Ästhetik des Performativen. Frankfurt a.M.: Suhrkamp

Hudelist, Andreas \& Krammer, Stefan (2017): Kultur des Performativen (Themenheft). In: Hudelist, Andreas \& Krammer, Stefan (Hrsg.):

Informationen zur Deutschdidaktik (IDE) 41/3

Goethe, Johann Wolfgang von (1795/96): Wilhelm Meisters Lehrjahre. In: Goethe. Berliner Ausgabe 10/1988. Poetische Werke. Romane und Erzählungen II. Berlin: Aufbau-Verlag

Mentz, Olivier \& Fleiner, Micha (Hrsg.) (2018): The Arts in Language Teaching. International Perspectives: Performative - Aesthetic - Transversal. Zürich: LIT Verlag

Schewe, Manfred (2013): Taking Stock and Looking Ahead: Drama Pedagogy as a Gateway to a Performative Teaching and Learning Culture. In: Scenario $\mathrm{VII} / 1,5-23$

Schiller, Friedrich (1879): An die Freunde. In: Schillers Sämmtliche Werke. Erster Band. Stuttgart: J.G. Cotta'sche Buchhandlung, 135-136. Online 
abrufbar unter: http://gutenberg.spiegel.de/buch/gedichte-9097/53

[letzter Zugriff: 27.12.2018] 